\title{
Effects of yogurt starter cultures on the survival of Lactobacillus acidophilus
}

\author{
Elizabeth W. Ng Marie Yeung Phillip S. Tong
}

\begin{abstract}
A B S T R A C T
Recognized to confer health benefits to consumers, probiotics such as Lactobacillus acidophilus are commonly incorporated into fermented dairy products worldwide; among which yogurt is a popular delivery vehicle. To materialize most of the putative health benefits associated with probiotics, an adequate amount of viable cells must be delivered at the time of consumption. However, the loss in their viabilities during refrigerated storage has been demonstrated previously. This study focused on the effects of yogurt starter cultures on the survival of five strains of $L$. acidophilus, with emphases on low $\mathrm{pH}$ and acid production. Differential survival behavior between $L$. acidophilus strains was further analyzed. To this end, viable cell counts of $L$. acidophilus were determined weekly during $4{ }^{\circ} \mathrm{C}$ storage in various types of yogurts made with Streptococcus thermophilus alone, L. delbrueckii ssp. bulgaricus alone, both species of the starter cultures, or glucono-delta-lactone (GDL). All yogurt types, except for pasteurized yogurts, were co-fermented with L. acidophilus. Yogurt filtrate was analyzed for the presence of any inhibitory substance and for the amount of hydrogen peroxide. Multiplication of $L$. acidophilus was not affected by the starter cultures as all strains reached high level on day 0 of the storage period. Throughout the 28-day storage period, cell counts of L. acidophilus PIM703 and SBT2062 remained steady $\left(\sim 6 \times 10^{7} \mathrm{CFU} / \mathrm{g}\right)$ in yogurts made with both starter cultures, whereas those of ATCC 700396 and NCFM were reduced by a maximum of 3 and 4.6 logs, respectively. When starter cultures were replaced by GDL, all strains survived well, suggesting that a low pH was not a critical factor dictating their survival. In addition, the filtrate collected from yogurts made with starter cultures appeared to have higher inhibitory activities against $L$. acidophilus than that made with GDL. The presence of viable starter cultures was necessary to adversely affect the survival of some strains, as pasteurized yogurts had no effect on their survival. In particular, the inhibitory effect exerted by L. delbrueckii ssp. bulgaricus on L. acidophilus NCFM was highly pronounced than by $S$. thermophilus, nevertheless, the same effect was not observed on SBT2062. The inhibition against stationary-phase NCFM cells might be caused by the elevated level of hydrogen peroxide produced by L. delbrueckii ssp. bulgaricus. Delineating factors driving the differences in survival trait among probiotic strains will lead to a more efficacious delivery of health benefits in fermented dairy products through targeted technological interventions.
\end{abstract}

\section{Introduction}

Fermented dairy products are commonly used as food vehicles to deliver probiotics to consumers. Among dairy products sold in the US, yogurt is likely the most recognized product containing probiotics (Lourens-Hattingh and Viljoen, 2001). As with many other countries, yogurts in the US are produced by pasteurized milk fermented by Streptococcus thermophilus and Lactobacillus delbrueckii ssp. bulgaricus. Probiotics are not required in the production, but are often added to carry out co-fermentation with the starter cultures.

"Probiotics" are defined as "live microorganisms which when administered in adequate amounts confer a health benefit on the host" (FAO/WHO, 2001). Numerous reports have suggested probiotics confer some forms of health benefits to humans. For instance, they have been shown in various extents to produce antimicrobial compounds, modulate host immune system, inhibit Helicobacter pylori, alleviate lactose intolerance, assimilate cholesterol, prevent autoimmunity, and exhibit antimutagenic properties (Adolfsson et al., 2004; Kailasapathy and Chin, 2000; McKinley, 2005; Parvez et al., 2006; Percival, 1997).

To realize most of these putative health benefits, it is understandable that a sufficient amount of viable probiotics must reach the intestines. Thus, along with the innate health-promoting capability, their viability in the products has been cited as an important prerequisite for achieving beneficial health effects (Galdeano and Perdigón, 2004). Hence, different forms of delivery vehicles should be studied and optimized to ensure that probiotics are viable and delivered in sufficient numbers before the expiration date (Godward et al., 2000). Based on previous studies characterizing a wide range of probiotic species and strain, a very high dose - minimum of $10^{8} \mathrm{CFU} /$ 
day, mostly in the range of $10^{10}-10^{11} \mathrm{CFU} /$ day - was required for the respective health benefits (Lourens-Hattingh and Viljoen, 2001; Parvez et al., 2006; Tamime et al., 2005). For instance, Lactobacillus acidophilus, a common probiotic species, was administered at a minimum level of $10^{9} \mathrm{CFU}$ daily to prevent or treat some gastrointestinal (GI) disorders (reviewed by WGO, 2008). Kailasapathy and Chin (2000) also suggested that the minimum therapeutic dose of probiotics should be $10^{8}$ to $10^{10} \mathrm{CFU} /$ day. This amount could be translated into $\geq 10^{6} \mathrm{CFU} / \mathrm{g} /$ day of probiotics-containing yogurt given that $100 \mathrm{~g}$ is the daily serving portion. High dosage is required to compensate for the loss of cells during the passage through the upper and lower parts of the GI tract (Tamime et al., 2005). For probiotics delivered through a food vehicle, additional amounts of cells are likely required prior to processing to account for the loss of cells during the processing and/or storage phases.

Maintaining a high level of viable probiotic cell count in yogurts throughout the shelf life, however, is not a simple task. Many factors influence the viability of probiotics in yogurts: strain variation, acid accumulation, interaction with starter cultures, level of dissolved oxygen and hydrogen peroxide $\left(\mathrm{H}_{2} \mathrm{O}_{2}\right)$, and storage condition (Donkor et al., 2006; Gilliland and Speck, 1977; Nighswonger et al., 1996; Talwalkar and Kailasapathy, 2003). Evidently, several studies reported that some commercially available dairy products contain insufficient number of viable probiotics (as defined by $<10^{6} \mathrm{CFU} / \mathrm{g}$ or $\mathrm{mL}$ before the expiration date), thereby diminishing the potential health benefits conferred by these products (Coeuret et al., 2004; Huys et al., 2006; Lin et al., 2006; Tharmaraj and Shah, 2003). Thus, understanding the survival of probiotics and developing methods to maintain and/or to promote their viability throughout the product shelf life continues to be an important subject of research in this field.

Many previous studies focused on devising strategies to improve the viability of particular L. acidophilus strains that showed suboptimal survival in yogurts (reviewed by Shah, 2000). These include reducing the oxygen content in the food by adding ascorbic acid (Dave and Shah, 1997a), and protecting the probiotics by means of encapsulation or addition of cryoprotectants (Capela et al., 2006). This study, on the other hand, undertook a comparative approach to probe the effects of acid and the presence of starter cultures on the survival of $L$. acidophilus in yogurts during the storage phase. This will help pinpoint the causes of suboptimal survival and provide a basis to develop more effective measures. Thus, the objectives were to determine the survival of five different $L$. acidophilus strains in yogurts made with different combinations of yogurt starter cultures, and to determine their survival independent of the starter culture fermentation by using an acidulant. The best and worst survival strains were chosen to further investigate factors attributing to the differential viabilities.

Enhancing our understanding of $L$. acidophilus survivability in yogurts may provide a foundation to improving probiotic strains and/ or starter cultures, and subsequently lead to a more effective delivery of probiotic-associated health benefits via fermented dairy products.

\section{Materials and methods}

\subsection{Bacterial strains and culture media}

Commercial starter cultures (YoFlex-L702), which contained S. thermophilus and L. delbrueckii ssp. bulgaricus, were provided by Chr. Hansen (Milwaukee, WI) in Direct Vat Set form. The isolation of these two species was made on MRS agar (BD Diagnostics, Sparks, MD). After incubation at $37{ }^{\circ} \mathrm{C}$ anaerobically for $48 \mathrm{~h}$, two distinct colony morphologies were observed. Each type was tested with a Gram stain kit (BD Diagnostics) to confirm their species identity - L. delbrueckii ssp. bulgaricus is gram positive bacillus whereas $S$. thermophilus is gram positive coccus. Each species was streaked for single colony isolation on M17 (S. thermophilus) or MRS (L. delbrueckii ssp. bulgaricus) medium (Ravula and Shah, 1998). After incubation at $37^{\circ} \mathrm{C}$ for $24-36 \mathrm{~h}$, MRS broth supplemented with $20 \%$ glycerol (Fisher Scientific, Tustin, CA) was used to make frozen stocks. Cultures were subcultured at least twice prior to all experiments.

Five strains of $L$. acidophilus were tested in this study. These strains were NCFM, ATCC 700396, PIM703, SBT2062 and LA-5. The first four strains were previously isolated from commercial products or obtained from reliable sources (Yeung et al., 2002), while LA-5 was kindly provided by Chr. Hansen. Their species identities were confirmed by API $50 \mathrm{CH}$ (bioMérieux, France) and partial 16S rDNA sequencing.

\subsection{Production of different types of yogurt}

An important aim of this study was to study the impact of the starter cultures on L. acidophilus. Therefore, yogurts with or without starter cultures were produced in the laboratory. As described below, the typical yogurt was made with the fermentation of both starter cultures (S. thermophilus and L. delbrueckii ssp. bulgaricus). Yogurts were also made with individual starter culture species. Finally, yogurts were made without any starter cultures. For all types of yogurt, 14\% (w/w) reconstituted low-heat nonfat dry milk (NFDM) (Dairy America, Inc., Fresno, CA) was used initially, and L. acidophilus was added immediately following the starter culture(s) or an acidulant.

For yogurts made with both starter cultures, a package of $250-\mathrm{g}$ YoFlex-L702 was thawed in a water bath at room temperature. Following the recommendation from the starter culture provider, it was then diluted 10 -fold with sterile $10 \%$ reconstituted NFDM, and $6 \mathrm{~mL}$ of this suspension was added into $3 \mathrm{~L}$ of pasteurized (heat treatment at $85^{\circ} \mathrm{C}$ for $30 \mathrm{~min}$ ) reconstituted NFDM being held at $43^{\circ} \mathrm{C}$. For yogurts made with individual species, $1 \%(\mathrm{v} / \mathrm{v})$ of the overnight-grown $S$. thermophilus or $L$. delbrueckii ssp. bulgaricus was inoculated into reconstituted NFDM. For yogurts made without any starter cultures, glucono-delta-lactone (GDL) was added at a concentration of $1.6 \%$ (w/ v). GDL is an acidulant that gradually lowers the $\mathrm{pH}$ of milk, mimicking the acid production of starter cultures. Therefore, the purpose of adding GDL was to study the effect of acid on L. acidophilus survival independent of any metabolic activity of starter cultures. The concentration of GDL was determined empirically to achieve similar fermentation time. Using a standard practice, all yogurts were not pasteurized post-fermentation except for one experiment. To prepare pasteurized yogurts, yogurts made with starter cultures or GDL was heated at $75{ }^{\circ} \mathrm{C}$ for $15 \mathrm{~min}$ prior to adding L. acidophilus NCFM or SBT2062 at a concentration of $5 \%(\mathrm{v} / \mathrm{v})$.

Except for the pasteurized yogurts and yogurts made for collecting filtrate (described in Sections 2.4 and 2.5), five strains of $L$. acidophilus were inoculated as separate samples immediately following the addition of starter culture(s) or GDL at a concentration of $5 \%(\mathrm{v} / \mathrm{v})$, which was determined to be $\sim 10^{6} \mathrm{CFU} / \mathrm{mL}$ by standard plate count (SPC). The $\mathrm{pH}$ of the mixtures was monitored periodically during the incubation at $43^{\circ} \mathrm{C}$. Incubation was ceased when $\mathrm{pH}$ reached 4.6 , which typically took $\sim 5 \mathrm{~h}$. Samples were immediately transferred to $4{ }^{\circ} \mathrm{C}$ (day 0 ) and stored for 28 days, the typical shelf life of commercial yogurts. Aliquots of each sample were removed from the refrigerator on days $0,7,14,21$ and 28 , and were subjected to enumeration of $L$. acidophilus and $\mathrm{pH}$ measurement. The experiments were carried out in triplicate. Throughout the entire refrigerated storage period (days 0 to 28 ), the $\mathrm{pH}$, ranging from 4.59 to 4.62 , was consistent for all types of yogurt including the one made without starter cultures.

\subsection{Enumeration of L. acidophilus in yogurt}

SPC, a conventional method to determine cell count, was used to quantify viable $L$. acidophilus cells. One gram of yogurt sample was diluted with $99 \mathrm{~mL}$ of sterile phosphate buffer saline (PBS), $\mathrm{pH} 7.2$ (Hardy Diagnostics, Santa Maria, CA). Subsequent 10-fold serial dilutions were made with PBS, and $0.1 \mathrm{~mL}$ of the diluted samples was spread on MRS-maltose agar, a selective medium for L. acidophilus 
(IDF, 1995). This medium was tested empirically prior to the experiments and was clearly able to selectively cultivate the five $L$. acidophilus strains amidst the presence of the starter cultures. After anaerobic incubation at $37{ }^{\circ} \mathrm{C}$ for $48-72 \mathrm{~h}, \mathrm{CFU} / \mathrm{g}$ was calculated. Relative survival for each strain was determined by dividing the CFU/g on days $7,14,21$ or 28 by the initial cell count on day 0 , and then multiplied by 100 . For all types of yogurts, the initial viable cell counts of L. acidophilus strains on day 0 ranged from $10^{7}$ to $10^{8} \mathrm{CFU} / \mathrm{g}$.

\subsection{Preparation of yogurt filtrate}

To study the effect of secretory metabolic products produced from starter cultures on probiotics, the survival of $L$. acidophilus NCFM was determined in MRS broth containing yogurt filtrate. Yogurt filtrate was prepared similarly as described by Dave and Shah (1998). Equal volume (in our experiments, $100 \mathrm{~mL}$ ) of sterile PBS, pH 7.2 (Hardy Diagnostics) was added into yogurt ( $100 \mathrm{~g}$ ) made with starter cultures or GDL, but free of $L$. acidophilus. The diluted yogurt mixture was prefiltered through a Whatman \#42 filter (Fisher Scientific) and then a cellulose acetate membrane with a pore size of $0.22 \mu \mathrm{m}$ (Fisher Scientific). Various amounts of sterile filtrate collected from these two types of yogurt were added into 30-mL MRS broth containing overnight-grown cultures of $L$. acidophilus NCFM. Enumeration of $L$ acidophilus NCFM, being incubated at $4{ }^{\circ} \mathrm{C}$, was performed for 15 days at 3-day intervals.

\subsection{Determination of hydrogen peroxide content}

An enzymatic method as described by Gilliland (1969) was used to measure the amount of $\mathrm{H}_{2} \mathrm{O}_{2}$ in yogurt on day 0 . Owing to the high viscosity of yogurt, the measurement was made on diluted yogurt filtrate. To prepare the filtrate, $10 \mathrm{~g}$ of yogurt was adjusted to $\mathrm{pH} 4.5$ with $0.1 \mathrm{~N} \mathrm{HCl}$. Two milliliters of $0.1 \mathrm{M}$ acetate buffer was added, and the final volume was brought up with sterile $\mathrm{dIH}_{2} \mathrm{O}$ to $20 \mathrm{~mL}$. Five milliliters of filtrate was collected following filtration through a Whatman \#42 filter (Fisher Scientific), and then transferred to a sterile glass tube containing $100 \mu \mathrm{L}$ of $1 \%$ o-dianisidine and $1 \mathrm{~mL}$ of $0.01 \mathrm{mg} / \mathrm{mL}$ horseradish peroxidase (HRP) (Fisher Scientific). Sample blank was prepared by replacing HRP with $\mathrm{dIH}_{2} \mathrm{O}$. After 10 min of incubation at room temperature, the reaction was stopped with the addition of $200 \mu \mathrm{L}$ of $4 \mathrm{~N} \mathrm{HCl}$. The absorbance of samples and sample blanks was measured at $400 \mathrm{~nm}$, and $\mathrm{H}_{2} \mathrm{O}_{2}$ content $(\mu \mathrm{g} / \mathrm{mL})$ was calculated by comparing to a standard curve.

\subsection{Statistical analysis}

To analyze the differences of $L$. acidophilus survival among samples, two-way analysis of variance (ANOVA) was conducted using the General Linear Model in Minitab software version 15.1 (State College, PA). All relative survival data were transformed using a based-10 logarithm (log relative survival), and a value of 0.00001 was added if values were zero. The log relative survival was the response variable, while the yogurt type and $L$. acidophilus strains were factors. Each replication was a block. Significant difference was determined with mean values comparison using Tukey test at $5 \%$ confidence $(P<0.05)$. One-way ANOVA was used to analyze the survival of $L$. acidophilus in various amounts of yogurt filtrate, and the amount of $\mathrm{H}_{2} \mathrm{O}_{2}$ produced by different samples. Statistical significance was defined as $P<0.05$.

\section{Results}

3.1. Survival of L. acidophilus in the presence and absence of starter cultures

In the presence of both starter cultures, the survival of some $L$. acidophilus strains was negatively affected (Fig. 1). Among the five $L$. acidophilus strains, the best and worst survival strain was SBT2062

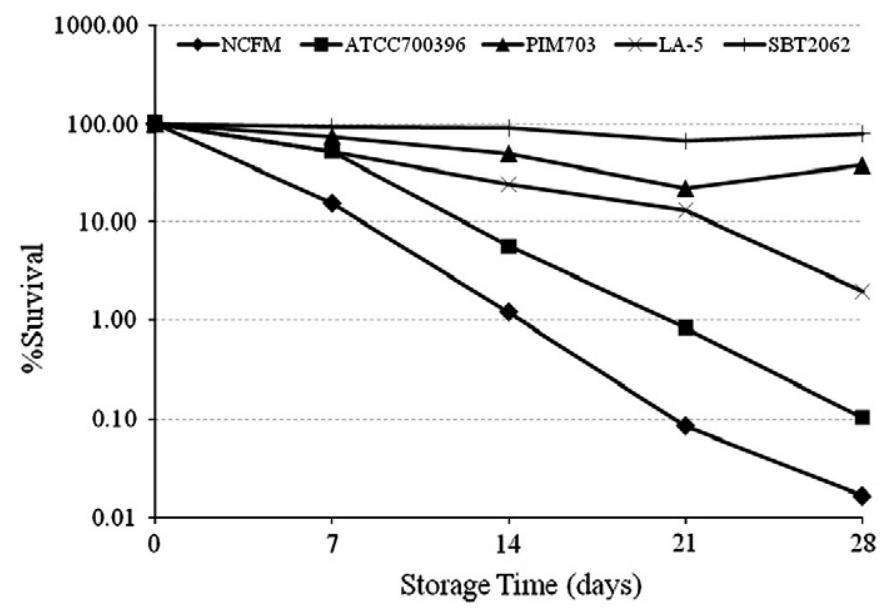

Fig. 1. Relative survival of five different L. acidophilus strains (NCFM [ ]; ATCC 700396

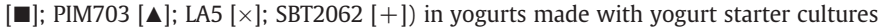
during 28 days of storage period at $4{ }^{\circ} \mathrm{C}$. Data shown are representatives of triplicate experiments.

and NCFM, showing a mean log reduction of 0.05 and 2.8, respectively. L. acidophilus ATCC 700396 also showed poor survival and statistically not different from NCFM. Viable cell counts of both NCFM and ATCC 700396 decreased drastically beginning on day 14 . Their final mean viable cell count on day 28 were $1.3 \times 10^{4}$ and $1.1 \times 10^{5} \mathrm{CFU} / \mathrm{g}$, respectively. Statistical analysis showed that the relative survival of L. acidophilus NCFM was significantly lowered than that of SBT2062, LA-5, and PIM703 $(P<0.05)$. On the other hand, when starter cultures were replaced with GDL, all strains exhibited good survival throughout the refrigerated storage period (Fig. 2) with only 0.81-log reduction for L. acidophilus NCFM.

\subsection{L. acidophilus survival in the presence of individual starter culture species}

To pinpoint if the negative impact was caused by a particular starter culture species, L. acidophilus SBT2062 and NCFM, representing the best and worst survival strains, were tested further in similar survival assays using yogurts made with either $S$. thermophilus alone or L. delbrueckii ssp. bulgaricus alone (Fig. 3). The latter species had a stronger effect on L. acidophilus NCFM, which showed a mean 2.5-log reduction $\left(4.0 \times 10^{7}\right.$ to $\left.1.3 \times 10^{5} \mathrm{CFU} / \mathrm{g}\right)$, compared to SBT2062 that

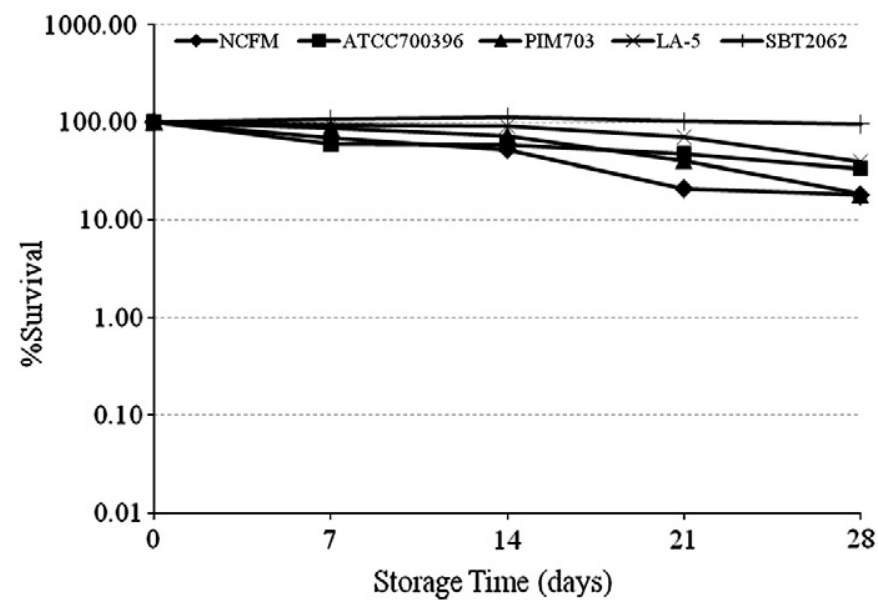

Fig. 2. Relative survival of five different L. acidophilus strains (NCFM [ $\$$ ]; ATCC 700396 [ ] ] PIM703 [ $\mathbf{\Delta}$ ]; LA5 [×]; SBT2062 [+]) in yogurts made with glucono-delta-lactone during 28 days of storage period at $4{ }^{\circ} \mathrm{C}$. Data shown are representatives of triplicate experiments. 


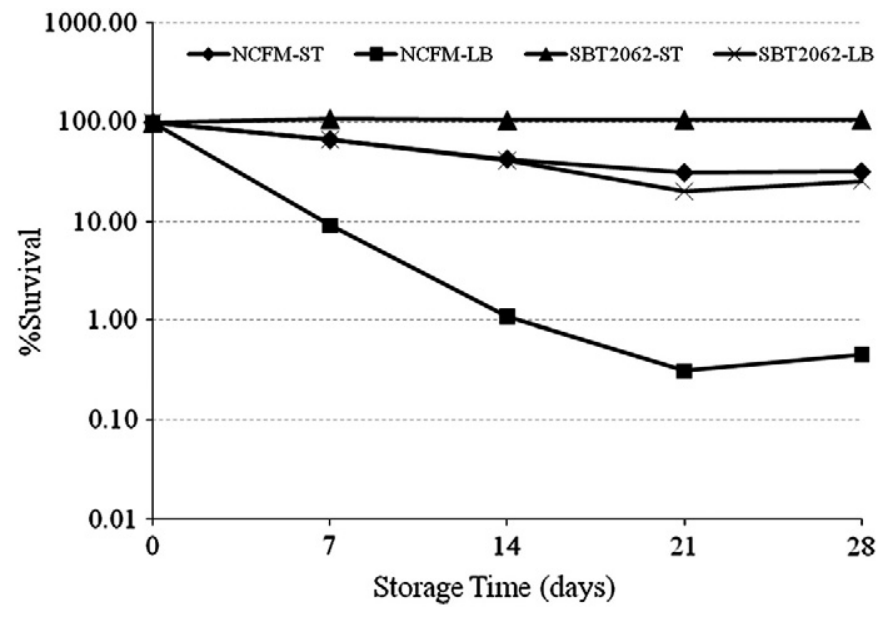

Fig. 3. Relative survival of $L$. acidophilus NCFM and SBT2062 in yogurts made with individual starter culture species, S. thermophilus or L. delbrueckii ssp. bulgaricus, during 28 days of storage period at $4{ }^{\circ} \mathrm{C}$. Data shown are representatives of triplicate experiments. (NCFM in yogurt made with S. thermophilus [ ]; with L. delbrueckii ssp.

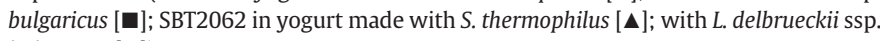
bulgaricus $[\times])$.

only had $0.6-\log$ reduction $\left(7.1 \times 10^{7}\right.$ to $\left.1.8 \times 10^{7} \mathrm{CFU} / \mathrm{g}\right)$. A marginal significant difference was observed at $P=0.0569$. On the other hand, NCFM survived well in yogurts made with $S$. thermophilus alone (0.5log reduction) while SBT2062 had 100\% survival. Comparing with the previous experiment, the antagonistic effect of yogurts made with both starter cultures on the survival of L. acidophilus NCFM appeared to be the summation of the antagonism exerted by the individual species.

\subsection{Starter cultures metabolites and L. acidophilus survival}

When known amounts of L. acidophilus NCFM and SBT2062 were added to pasteurized yogurts made with starter cultures or GDL, both strains showed steady survival with only a maximum of $0.15-\log$ reduction $\left(1.4 \times 10^{7}\right.$ to $\left.9.9 \times 10^{6} \mathrm{CFU} / \mathrm{g}\right)$ during a 15 -day storage period for NCFM (Fig. 4). To investigate if these two strains were particularly susceptible to any thermolabile secretory metabolites that might have been produced by the starter cultures but were

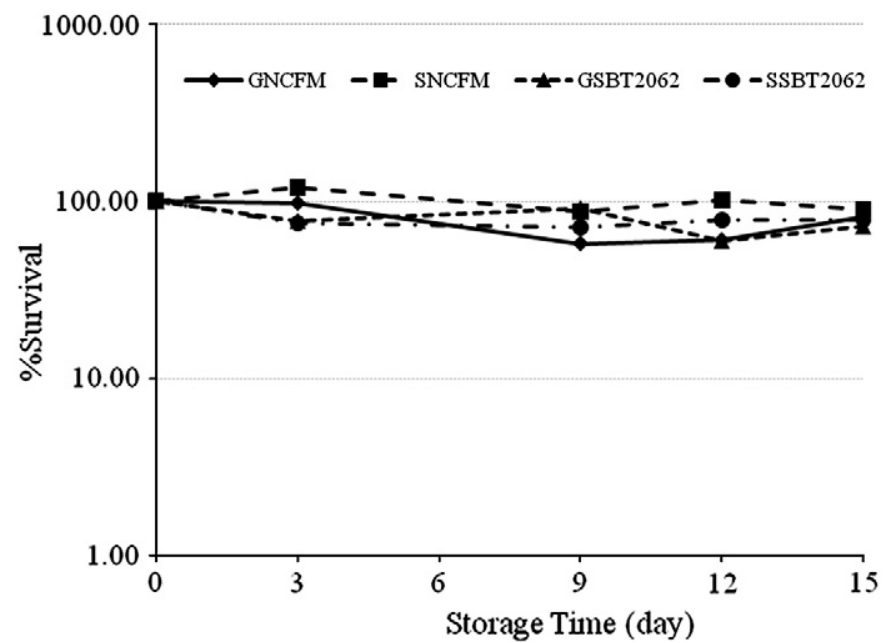

Fig. 4. Relative survival of $L$. acidophilus NCFM and SBT2062 in pasteurized yogurts made with starter cultures or glucono-delta-lactone (GDL) during 15 days of storage period at $4{ }^{\circ} \mathrm{C}$. (NCFM in pasteurized yogurts made with GDL [GNCFM, $\bullet$ ]; with starter cultures [SNCFM, - ]; SBT2062 in pasteurized yogurts made with GDL [GSBT2062, $\mathbf{\Delta}$ ]; with starter cultures [SSBT2062, @]). inactivated in the pasteurization step, a preliminary assay based on agar diffusion was conducted. No obvious inhibition zone around $S$. thermophilus or L. delbrueckii ssp. bulgaricus against L. acidophilus NCFM and SBT2062 was observed (data not shown). As we speculated this assay might not be sensitive enough, yogurt filtrate was tested in a subsequent experiment. As shown in Table 1, different ratios of filtrate collected from yogurts made with starter cultures or GDL were added into fresh MRS containing known amounts of L. acidophilus NCFM. Among the treatments, differences in cell count at various time points were not statistically significant. Nevertheless, an interesting trend emerged - after day 6, the survival of L. acidophilus NCFM was reduced to a greater extent in the presence of a higher concentration of yogurt filtrate made with starter cultures than with GDL.

The level of $\mathrm{H}_{2} \mathrm{O}_{2}$ in various types of yogurts was measured to probe its association with $L$. acidophilus survival. Yogurt prepared with L. delbrueckii ssp. bulgaricus alone yielded seven- to nine-fold higher amounts of $\mathrm{H}_{2} \mathrm{O}_{2}$ than those prepared with $\mathrm{S}$. thermophilus alone or with both species (Fig. 5). Levels of $\mathrm{H}_{2} \mathrm{O}_{2}$ in pre- and post-pasteurized reconstituted NFDM were relatively low.

\section{Discussion}

Utilizing food as a vehicle to deliver probiotics has been an interest to food scientists for decades due to their purported health-associated benefits to customers. Maintaining viable probiotic cell counts at high level by the end of the expiration date is required for most health benefits. Nevertheless, it has been a challenge since probiotics are subjected to various environmental stresses during food production and storage, thereby decreasing their numbers. The identification of factors negatively affecting the survival of probiotics may lead to the development of strain improvement strategy, food processing optimization, and evaluation of viability-promoting substances. This study aimed to address the effects of starter cultures on $L$. acidophilus and used a comparative approach to help understand the differential survival behavior observed between strains.

As with the intrinsic probiotic activities, the survival behavior of $L$. acidophilus in yogurts during refrigerated storage period also displayed significant strain-to-strain variation. Though the growth of all $L$. acidophilus strains examined was not apparently affected, the survival of some strains was clearly hampered in the presence of yogurt starter cultures. During our assessment on how starter cultures affected L. acidophilus viability, a treatment without starter cultures must be included for comparison. To eliminate $\mathrm{pH}$ as a confounding variable, commercially available GDL was added as an acidulant to yield the same $\mathrm{pH}$ value post-fermentation as the other yogurt types. GDL releases gluconic acid gradually at a rate comparable to organic acids produced by the starter cultures. It has a pKa of 3.6, proximate to that of lactic acid ( $\mathrm{pKa}=3.8$ ) predominately produced by the starter cultures. All L. acidophilus strains exhibited good survival, suggesting

Table 1

Amount of yogurt filtrate added into fresh MRS and the survival of L. acidophilus NCFM during refrigerated storage. Survival on day 0 is normalized to $100 \%$.

\begin{tabular}{|c|c|c|c|c|c|c|}
\hline \multicolumn{2}{|c|}{$\begin{array}{l}\text { Amount of } \\
\text { yogurt } \\
\text { filtrate }(\mathrm{mL})\end{array}$} & \multicolumn{5}{|l|}{ Survival (\%) } \\
\hline STLB $^{\mathrm{a}}$ & $\overline{\mathrm{GDL}^{\mathrm{b}}}$ & Day 3 & Day 6 & Day 9 & Day 12 & Day 15 \\
\hline 0 & 10 & $82.9 \pm 1.2$ & $37.9 \pm 7.5$ & $27.50 \pm 3.54$ & $20.4 \pm 4.4$ & $16.5 \pm 5.5$ \\
\hline 1 & 9 & $83.5 \pm 8.5$ & $39.3 \pm 0.9$ & $32.2 \pm 10.7$ & $24.8 \pm 9.6$ & $18.5 \pm 9.7$ \\
\hline 5 & 5 & $105.8 \pm 24.2$ & $26.6 \pm 15.3$ & $16.1 \pm 8.8$ & $12.2 \pm 9.9$ & $11.9 \pm 10.1$ \\
\hline 9 & 1 & $73.3 \pm 3.0$ & $21.4 \pm 5.0$ & $18.1 \pm 1.1$ & $15.1 \pm 1.2$ & $11.2 \pm 2.4$ \\
\hline 10 & 0 & $89.3 \pm 7.9$ & $21.8 \pm 2.9$ & $17.7 \pm 4.0$ & $13.0 \pm 1.4$ & $10.4 \pm 1.5$ \\
\hline
\end{tabular}

a STLB is the yogurt filtrate originated from yogurts made with starter cultures.

b GDL is the yogurts filtrate originated from yogurt made with glucono-delta-lactone. 


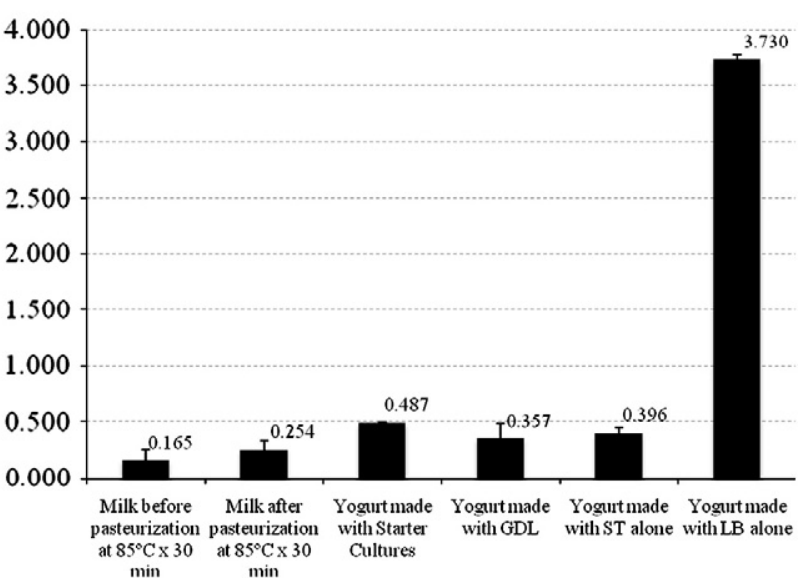

Fig. 5. Hydrogen peroxide content $(\mu \mathrm{g} / \mathrm{mL})$ in pre- and post-pasteurized reconstituted non-fat dry milk, and different types of yogurt. S. thermophilus and L. delbueckii ssp. bulgaricus are abbreviated as [ST] and [LB], respectively.

that the low pH due to gluconic acid accumulation was not a critical factor influencing their viabilities. Gilliland and Speck (1977) also showed $L$. acidophilus NCFM survived well at $5{ }^{\circ} \mathrm{C}$ for 14 days in $10 \%$ non-fat milk solid adjusted to $\mathrm{pH} 4.2$ with lactic acid. In one of our experiments, yogurts made with $S$. thermophilus or L. delbrueckii ssp. bulgaricus alone also did not produce significant effect on the survival of $L$. acidophilus SBT2062, further suggesting that organic acids produced by starter cultures did not necessarily impair the survival of all probiotics.

Our findings appear to contradict some previous studies that reported low $\mathrm{pH}$ and organic acids accumulation were amongst the important factors contributing to the loss of cell viability of probiotics (Donkor et al., 2006; Kailasapathy et al., 2007; Vinderola et al., 2002). For instance, Kailasapathy et al. (2007) studied the survival of $L$. acidophilus LAFTI ${ }^{\circledR}$ L10 in stirred fruit yogurts and suggested that the $\mathrm{pH}$ from the fruit preparation influenced the strain's viability. Donkor et al. (2006) also concluded that the decrease in L. acidophilus cell numbers in yogurts was the result of lactic and acetic acids accumulation. Interestingly, in this previous study, the level of organic acids was found to have a more pronounced effect on the strain's variability than the $\mathrm{pH}$ value. Indeed, these organic acids have higher pKa values than the strong acids like hydrochloric acid $(\mathrm{HCl})$ and thus greater proportions are in the undissociated form, facilitating them to translocate across the cell membrane. For this reason, lactate, acetate and other volatile acids are commonly used as preservatives. This explanation is consistent with the findings of Azcarate-Peril et al. (2004) that showed L. acidophilus NCK1398 drastically lost its viability in MRS broth acidified with lactic acid, but not with $\mathrm{HCl}$.

This study, nonetheless, showed that our five L. acidophilus strains demonstrated an effective $\mathrm{pH}$ homeostasis mechanism when exposed to organic acid accumulation due to GDL. As mentioned, L. acidophilus SBT2062 was also able to resist other types of acid accumulation caused by $S$. thermophilus and L. delbrueckii ssp. bulgaricus. According to previous studies, $L$. acidophilus possesses several mechanisms to counteract acid stress (Altermann et al., 2005; Azcarate-Peril et al., 2004; Lorca and de Valdez 2001a, 2001b). Some have high cytoplasmic buffering capacity $(\mathrm{pH} 3.72-7.73$ ) that may increase their stability and allow them to resist wider changes in the intracellular $\mathrm{pH}\left(\mathrm{pH}_{\mathrm{i}}\right)$ under acidic conditions (Rius et al., 1994). In the presence of organic acids, the $\mathrm{F}_{1} \mathrm{~F}_{0}$-ATPase system helps maintain the $\mathrm{pH}_{\mathrm{i}}$. The inducible $\mathrm{pH}$ homeostasis system, mediated by a protontranslocating ATPase as a mechanism to increase the $\mathrm{pH}_{\mathrm{i}}$, might be an important mechanism in the success of $L$. acidophilus such as NCFM to survive low pH environment (Kullen and Klaenhammer, 1999). Elucidated from sequence analyses followed by mutagenesis experiments, L. acidophilus NCFM appears to also utilize amino acid decarboxylation as a means to increase $\mathrm{pH}_{\mathrm{i}}$ (Azcarate-Peril et al., 2004). Some $L$. acidophilus strains have shown to mount an effective acid tolerance response in which the pre-exposure of cultures to a less severe $\mathrm{pH}$ stress such as $\mathrm{pH}$ 5.0-5.5 triggered a more effective mechanism to offset a more severe acid stress such as $\mathrm{pH}$ 3.0-3.5 (Azcarate-Peril et al., 2004; Lorca et al., 2002).

While low $\mathrm{pH}$ (4.6) and GDL accumulation had no effect on the survival of $L$. acidophilus strains, some (especially NCFM) were strongly influenced by the presence of starter cultures. Experiments using pasteurized yogurts showed that killed starter cultures did not affect the survival of $L$. acidophilus NCFM and SBT2062. Thus, the presence of viable starter cultures or their thermostable metabolites was likely a necessary factor. Based on this hypothesis, we had expected that the filtrate collected from yogurt made with GDL would not influence the survival of $L$. acidophilus NCFM and SBT2062, while that collected from yogurts made with starter cultures would. However, the survival of both strains was impacted by both types of yogurt filtrates, possibly due to the diluted nutrient in MRS. The concentration of the secretory metabolites, if present, may also be too low to produce a discernable difference. Though statistically insignificant, the trend suggested that at least one starter culture species secreted some antagonistic metabolites against $L$. acidophilus NCFM.

Results from the experiments using yogurts made with individual starter culture species provided evidence that the presence of $L$. delbrueckii ssp. bulgaricus was more inhibitory to L. acidophilus. The differential impact of the individual starter culture species on $L$. acidophilus SBT2062 and NCFM led us to speculate that the latter strain is a poor competitor when viable L. delbrueckii ssp. bulgaricus or its metabolites is present. Several groups also demonstrated the instability of L. acidophilus in yogurts made with L. delbrueckii ssp. bulgaricus alone. Therefore, the exclusion of this species in yogurt production has been suggested to eliminate the antagonistic effect (Gilliland and Speck, 1977; Lourens-Hattingh and Viljoen, 2001).

While the main objective of this study was not sought to identify inhibitory metabolites, we believe further analyses might confirm previous findings and/or provide a new perspective. To this end, we chose to investigate the relationship between $\mathrm{H}_{2} \mathrm{O}_{2}$ and L. acidophilus viability. We did not test for the presence of bacteriocin based on the negative screening results according to the agar diffusion assay. We also did not focus on the packaging materials and the amount of dissolved oxygen in yogurt, which were suggested by some as important factors (Shah, 2000), as they were unlikely significant variables in this study in which the same type of container, and same processing and storage conditions were employed across all experiments.

Corroborating previous studies (Dave and Shah, 1997b; LourensHattingh and Viljoen, 2001; Tamime et al., 2005), the presence of $L$. delbrueckii ssp. bulgaricus in yogurts yielded high level of $\mathrm{H}_{2} \mathrm{O}_{2}$. Dave and Shah (1997b) reported that yogurts prepared with $L$. delbrueckii ssp. bulgaricus, L. acidophilus and bifidobacteria had 6-6.5 $\mu \mathrm{g} / \mathrm{g}$ of $\mathrm{H}_{2} \mathrm{O}_{2}$ immediately post-fermentation compared to $2.5 \mu \mathrm{g} / \mathrm{g}$ for those prepared with $S$. thermophilus in lieu of $L$. delbrueckii ssp. bulgaricus. It is perceivable that $L$. delbrueckii ssp. bulgaricus generates $\mathrm{H}_{2} \mathrm{O}_{2}$ primarily due to the action of NADH: $\mathrm{H}_{2} \mathrm{O}_{2}$ oxidase (Marty-Teysset et al., 2000; Sakamoto and Komagata, 1996), while the presence of $S$. thermophilus helps decompose $\mathrm{H}_{2} \mathrm{O}_{2}$ via NADH peroxidase (Smart and Thomas, 1987), an enzyme converting $\mathrm{H}_{2} \mathrm{O}_{2}$ into water to regenerate $\mathrm{NAD}^{+}$.

Without sufficient scavenging mechanism, the intracellular accumulation of $\mathrm{H}_{2} \mathrm{O}_{2}$ and other toxic oxygenic metabolites such as superoxide anion and hydroxyl radical can eventually lead to microbial cell death (Talwalkar and Kailasapathy, 2003; Villegas and Gilliland, 1998). Gilliland and Speck (1977) studied the survival of $L$. 
acidophilus NCFM, CNRZ 216, and 4692 in yogurts by mixing them after the starter cultures fermentation. They concluded that $\mathrm{H}_{2} \mathrm{O}_{2}$ produced during the storage of yogurts was likely the main substance responsible for the loss in L. acidophilus viabilities. However, they did not study the effect of stirring, which inevitably introduced variable amounts of oxygen to the viscous mixture, on probiotic survival. In general, the $L$. acidophilus species does not possess catalase, an enzyme hydrolyzing $\mathrm{H}_{2} \mathrm{O}_{2}$ into water and oxygen. Thus, intentionally added catalase could improve the viability of $L$. acidophilus (Gilliland and Speck, 1977). Taking our data and previous findings into consideration, $\mathrm{H}_{2} \mathrm{O}_{2}$ might be a critical factor impairing the survival of $L$. acidophilus NCFM, but not SBT2062. The effect on NCFM, nevertheless, was only apparent in the survival or stationary phase.

To scavenge $\mathrm{H}_{2} \mathrm{O}_{2}$, certain L. acidophilus strains such as 2400 and 2409 produced NADH peroxidase that likely accounted for their good survival in very high concentrations (up to $30 \mathrm{mg} / \mathrm{g}$ ) of $\mathrm{H}_{2} \mathrm{O}_{2}$ (Talwalkar and Kailasapathy, 2003). This enzyme functions optimally at $\mathrm{pH} 5$, but its activity markedly drops at $\mathrm{pH} 4.5$. Since the $\mathrm{pH}$ of yogurts in the present study was 4.6, NADH peroxidase, if produced by the L. acidophilus strains, might not be highly active during the storage period. Nevertheless, it may be a starting point to study the production and activity of NADH peroxidase in L. acidophilus NCFM versus SBT2062 to better understand the differential survival behavior between these strains.

Another probable cause leading to the poor survival of NCFM was that its overall fitness may be somewhat deficient. Molecular analyses on gene and protein expressions between these strains, in relationship to their survival in dairy products, would be the next logical step to provide a more global picture and to pinpoint the mechanism of good (or poor) survival.

\section{Conclusion}

To capitalize the potential health benefits of probiotic L. acidophilus, they must be delivered at a high dose through food vehicles such as yogurts. While many previous studies reported that acid accumulation negatively affected their survival during the refrigerated storage period, we demonstrated low $\mathrm{pH}$ and/or organic acid accumulation did not necessarily play a critical role. Between the two starter culture species, $L$. delbrueckii ssp. bulgaricus exerted a greater detrimental effect on the survival of some $L$. acidophilus strains perhaps by producing inhibitory metabolites such as $\mathrm{H}_{2} \mathrm{O}_{2}$. As with their intrinsic probiotic activities, the viabilities of $L$. acidophilus in yogurts showed marked strain-to-strain variations. Comparing strains with varying survival behaviors may lead to the identification of new factors associated with better survival, development of processing/ storage strategies to enhance survival of probiotics, and subsequently more effective delivery of health benefits via fermented dairy products.

\section{Acknowledgements}

We are grateful to Rafael Jimenez and Anya Goodman of California Polytechnic State University for their valuable advice to this study. We also appreciate the California Dairy Research Foundation and Dairy Management Inc. for their continuing support.

\section{References}

Adolfsson, O., Meydani, S.N., Russell, R.M., 2004. Yogurt and gut function. The American Journal of Clinical Nutrition 80, 245-256.

Altermann, E., Russell, W.M., Azcarate-Peril, M.A., Barrangou, R., Buck, B.L., McAuliffe, O. Souther, N., Dobson, A., Duong, T., Callanan, M., Lick, S., Hamrick, A., Cano, R., Klaenhammer, T.R., 2005. Complete genome sequence of the probiotic lactic acid bacterium Lactobacillus acidophilus NCFM. Proceedings of the National Academy of Sciences USA 102, 3906-3912.
Azcarate-Peril, M.A., Altermann, E., Hoover-Fitzula, R.L., Cano, R., Klaenhammer, T.R. 2004. Identification and inactivation of genetic loci involved with Lactobacillus acidophilus acid tolerance. Applied and Environmental Microbiology 70, 5315-5322.

Capela, P., Hay, T.K.C., Shah, N.P., 2006. Effect of cryoprotectants, prebiotics and microencapsulation on survival of probiotic organisms in yoghurt and freeze-dried yoghurt. Food Research International 39, 203-211.

Coeuret, V., Gueguen, M., Vernoux, J.P., 2004. Numbers and strains of lactobacilli in some probiotic products. International Journal of Food Microbiology 97, 147-156.

Dave, R.I., Shah, N.P., 1997a. Viability of yogurt and probiotic bacteria in yoghurts made from commercial starter cultures. International Dairy Journal 7, 31-41.

Dave, R.I., Shah, N.P., 1997b. Effectiveness of ascorbic acid as an oxygen scavenger in improving viability of probiotic bacteria in yoghurts made with commercial starter cultures. International Dairy Journal 7, 435-443.

Dave, R.I., Shah, N.P., 1998. Ingredient supplementation effects on viability of probiotic bacteria in yogurt. Journal of Dairy Science 81, 2804-2816.

Donkor, O.N., Henriksson, A., Vasiljevic, T., Shah, N.P., 2006. Effect of acidification on the activity of probiotics in yoghurt during cold storage. International Dairy Journal 16 , 1181-1189.

Food, Agriculture Organization/World Health Organization (FAO/WHO), 2001. Health and nutritional properties of probiotics in food including powder milk with live lactic acid bacteria. Report of A Joint FAO/WHO Expert Consultation on Evaluation of Health and Nutritional Properties in Food Including Powder Milk with Live Lactic Acid Bacteria. Cordoba, Argentina.

Galdeano, C.M., Perdigón, G., 2004. Role of viability of ptobiotic strains in their persistence in the gut and in mucosal immune stimulation. Journal of Applied Microbiology 97, 673-681.

Gilliland, S.E., 1969. Enzymatic determination of residual hydrogen peroxide in milk. Journal of Dairy Science 52, 321-324.

Gilliland, S.E., Speck, M.L., 1977. Instability of Lactobacillus acidophilus in yogurt. Journal of Dairy Science 60, 1394-1398.

Godward, G., Sultana, K., Kailasapathy, K., Peiris, P., Arumugaswamy, R., Reynolds, N. 2000. The importance of strain selection on the viability and survival or probiotics bacteria in dairy foods. Milchwissenschaft 55, 441-445.

Huys, G., Vancanneyt, M., D'Haene, K., Vankerckhoven, V., Goossens, H., Swings, J., 2006 Accuracy of species identity of commercial bacterial cultures intended for probiotic or nutritional use. Research in Microbiology 157, 803-810.

International Dairy Federation (IDF), 1995. Detection and Enumeration of Lactobacillus acidophilus. Bulletin No. 306/1995.

Kailasapathy, K., Chin, J., 2000. Survival and therapeutic potential of probiotic organism with reference to Lactobacillus acidophilus and Bifidobacterium ssp. Immunology and Cell Biology 78, 80-88.

Kailasapathy, K., Harmstorf, I., Phillips, M., 2007. Survival of Lactobacillus acidophilus and Bifidobacterium animalis ssp. lactis in stirred fruit yogurts. LWT - Food Science and Technology 41, 1317-1322.

Kullen, M., Klaenhammer, T.R., 1999. Identification of the pH-inducible, protontranslocating F1F0-ATPase (atpBEFHAGDC) operon of Lactobacillus acidophilus by differential display: gene structure, cloning and characterization. Molecular Microbiology 33, 1152-1161.

Lin, W.H., Hwang, C.F., Chen, L.W., Tsen, H.Y., 2006. Viable counts, characteristic evaluation for commercial lactic acid bacteria products. Food Microbiology 23, 74-81.

Lorca, G.L., de Valdez, G.F., 2001a. A low-pH inducible, stationary-phase acid tolerance response in Lactobacillus acidophilus CRL639. Current Microbiology 42, 21-25.

Lorca, G.L., de Valdez, G.F., 2001b. Acid tolerance mediated by membrane ATPases in Lactobacillus acidophilus. Biotechnology Letters 23, 777-780.

Lorca, G.L., de Valdez, G.F., Ljungh, A., 2002. Characterization of the protein-synthesis dependent adaptive acid tolerance response in Lactobacillus acidophilus. Journal of Molecular Microbiology and Biotechnology 4, 525-532.

Lourens-Hattingh, A., Viljoen, B.C., 2001. Yoghurt as probiotic carrier food. International Dairy Journal 11, 1-17.

Marty-Teysset, C., de la Torre, F., Garel, J.-R., 2000. Increased production of hydrogen peroxide by Lactobacillus delbrueckii subsp. bulgaricus upon aeration: involvement of an NADH oxidase in oxidative stress. Applied and Environmental Microbiology 66, 262-267.

McKinley, M.C., 2005. The nutrition and health benefits of yoghurt. International Journal of Dairy Technology 58, 1-12.

Nighswonger, B.D., Brashears, M.M., Gilliland, S.E., 1996. Viability of Lactobacillus acidophilus and Lactobacillus casei in fermented milk products during refrigerated storage. Journal of Dairy Science 79, 212-219.

Parvez, S., Malik, K.A., Ah Kang, S., Kim, H.-Y., 2006. Probiotic and their fermented food products are beneficial for health. Journal of Applied Microbiology 100, 1171-1185.

Percival, M., 1997. Choosing a probiotic supplement. Clinical Nutrition Insights 6, 1-4.

Ravula, R.R., Shah, N.P., 1998. Selective enumeration of Lactobacillus casei from yogurts and fermented milk drinks. Biotechnology Techniques 12, 819-822.

Rius, N., So1é, M., Francia, A., Lorén, J.-G., 1994. Buffering capacity and membrane $\mathrm{H}^{+}$ conductance of lactic acid bacteria. FEMS Microbiology Letters 120, 291-296.

Sakamoto, M., Komagata, K., 1996. Aerobic growth of and activities of NADH oxidase and NADH peroxidase in lactic acid bacteria. Journal of Fermentation and Bioengineering 82, 210-216.

Shah, N.P., 2000. Probiotic bacteria: selective enumeration and survival in dairy foods. Journal of Dairy Science 83, 894-907.

Smart, J.B., Thomas, T.D., 1987. Effect of oxygen on lactose metabolism in lactic streptococci. Applied and Environmental Microbiology 53, 533-540.

Talwalkar, A., Kailasapathy, K., 2003. Metabolic and biochemical responses of probiotics bacteria in oxygen. Journal of Dairy Science 86, 2537-2546. 
Tamime, A.Y., Saarela, M., Søndergaard, A.K., Mistry, V.V., Shah, N.P., 2005. In: Tamime, A.Y. (Ed.), Production and maintenance of viability of probiotic microorganisms in dairy products. : Probiotic Dairy Products. Blackwell Publishing Ltd., Oxford, UK, pp. 39-72.

Tharmaraj, N., Shah, N.P., 2003. Selective enumeration of Lactobacillus delbrueckii ssp. bulgaricus, Streptococcus thermophilus, Lactobacillus acidophilus, Bifidobacteria, Lactobacillus casei, Lactobacillus rhamnosus, and Propionibacteria. Journal of Dairy Science 86, 2288-2296.

Villegas, E., Gilliland, S.E., 1998. Hydrogen peroxide production by Lactobacillus delbrueckii ssp. lactis at $5{ }^{\circ} \mathrm{C}$. Journal of Food Science 63, 1070-1074.
Vinderola, C.G., Mocchiutti, P., Reinheimer, J.A., 2002. Interaction among lactic acid starter and probiotic bacteria used for fermented dairy products. Journal of Dairy Science 85, 721-729.

World Gastroenterology Organisation (WGO), 2008. Practice guideline. Probiotics and Prebiotics. May.

Yeung, P.S.M., Sanders, M.E., Kitts, C.L., Cano, R., Tong, P.S., 2002. Species-specific identification of commercial probiotic strains. Journal of Dairy Science 85, 1039-1051. 